\title{
Merging Scored Bounding Boxes with Gaussian Mixture Model for Object Detection
}

\author{
Chunzhi Gu ${ }^{\mathrm{a}}$, Takumi Nakane ${ }^{\mathrm{a}}$, Takuya Akashi ${ }^{\mathrm{b}}$, Chao Zhang ${ }^{\mathrm{a}, *}$ \\ ${ }^{\mathrm{a}}$ University of Fukui, 3-9-1, Bunkyo, Fukui, 910-8507, Japan \\ ${ }^{\mathrm{b}}$ Iwate University, 4-3-5, Ueda, Morioka, 020-8551, Japan \\ *Corresponding Author: zhang@u-fukui.ac.jp
}

\begin{abstract}
Object detection has been struggled with the is sue that how to localize the accurate position of a target from a large number of scored detections. One of the most widely used methods is non-maximu m suppression (NMS). However, the fact that this method can only select the high-score detections locally makes the result sometimes less accurate. In this paper, we propose a novel approach to merge all the scored bounding boxes by Gaussian Mixture Model (GMM) that takes not only the spatial information but also the score of each detection into account. We report experiments on both tasks of pedestrian detection and face detection with publicly available datasets. The drawbacks of NMS can be overcome to some extent and the proposed method outperforms other conventional methods.
\end{abstract}

Keywords : Bounding box grouping, Gaussian Mixture Model, EM- algorithm, Clustering, Classification.

\section{Introduction}

Most of the object detection ${ }^{(1)(2)}$ methods rely on sliding windows ${ }^{(3)}$ from left top to right down for searching. Each candidate window will be sent to a classifier/detector which returns a score depending on how much confidence the classifier has to classify the window as positive. Usually, this procedure produces a large number of detections whose scores increase when getting closer to the correct location of the target. What the users are interested in most is only the window which tightly encloses the target, rather than the other numerous raw scored detections (i.e. we need to merge all the detections to, ideally, one bounding box (BB) per object).

The process of merging detections usually exploits non-maximu m suppression ${ }^{(4)}$ (NMS). The steps of merging

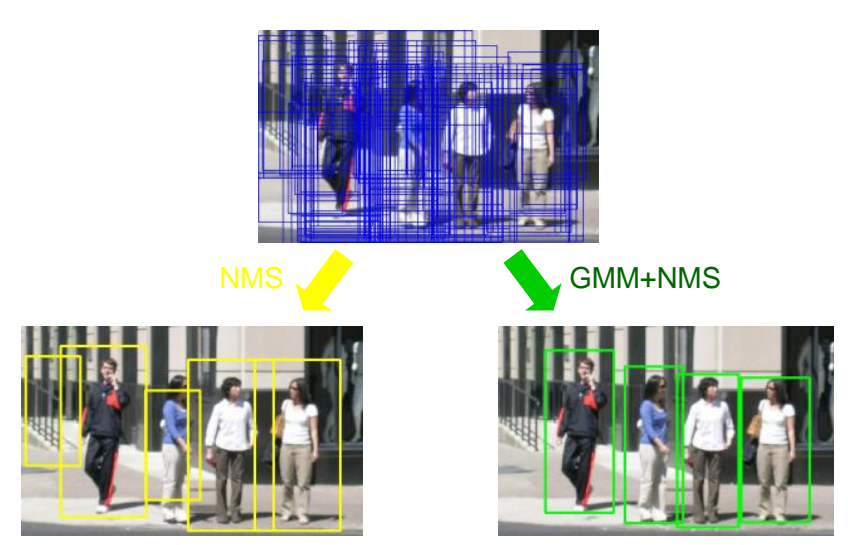

Fig. 1 An example of merging all raw detections. Raw detections (top), the results of NMS (bottom left), the results of the proposed method (bottom right) are shown respectively. Obviously, the results of NMS are not the best bounding boxes visually since they are either too large or sometimes too small. On the contrary, our proposed method takes both spatial in formation and score of each detection into account, which leads to better results.

detections with NMS is as follows:

1. Select the best-scored window among all of the detections.

2. Delete redundant detections according to a predetermined threshold.

3. Repeat 1-2 until there is no more BB which needs to be suppressed.

In the second step, the threshold controls how much the suppression is. Fast and simple though NMS is, the accuracy largely suffers from false positives. On the other hand, both accuracy and processing time depend on the sampling step when conducting sliding window. It is not that any classifier can reach an ideal accuracy that the closer the sliding window is to the target, the score for it is larger. With false positive showing up, the precision of NMS will decrease to a great extent because it only chooses 
the bounding box of the highest score. Also, it is time consuming to conduct sliding window at the step of one pixel. On the other hand, sampling BB with a relatively large step will inevitably cause the situation that the $\mathrm{BB}$ of the best location is not sampled, as a result of which the nearby BBs become the best choices. In conclusion, considering the characteristic of NMS, the top-scored BB may not be the ideal choice.

It is necessary to get over this drawback resulting from the limitation of NMS. Since the problem of merg ing all the detections can be interpreted as the clustering proble ${ }^{(5)}$, we utilize Gaussian Mixture Model (GMM) ${ }^{(6-7)}$ to cope with the merg ing problem.

The widely used clustering methods are mean-shift ${ }^{(8-10)}$ and K-means ${ }^{(11-13)}$. However, mean-shift depends on sufficient high-density data. Also, data to be clustered is much likely to fall into the wrong cluster when the data is relatively natural. As for K-means, it is generally incapable to find non-convex clusters, which lowers its versatility in various forms of data. A different idea is to formulate a probabilistic distribution to cluster the detections, which is proposed in ${ }^{(14)}$. However, the scale-sensitive Gaussians (SSG) in his work is a 3-d model with position and scale. The method neglects the potentially essential parameters (e.g. score, an isotropic scaling) of detections, and only sticks to the uniform scaling (one parameter) and location of BB (two parameters), which turns out to be inadequate.

In the case of GMM, all the detections obtained from the detector can be considered to comply with a kind of probabilistic distribution, and what we have contributed in this paper is to use a mixture of Gaussian distributions to approach the distribution of $B B$ 's parameters. Since each BB has 5 parameters, the proposed approach GMM is five dimensional. Nevertheless, GMM needs a pre-fixed $k$ for the number of clusters. In this paper, the $k$ is determined by the number of NMS's output. However, NMS cannot always perform accurately as we mentioned above, and it empirically tends to provide redundant results. To overcome this problem, we add an additional step to prune the results fro m GMM by reapplying NMS.

\section{Proposed method}

In this section, we introduce how we exploit the GMM and $\mathrm{EM}$ algorithm to estimate the parameters of BBs in order to merge the detections.

\subsection{Gaussian Mixture Model}

The probability density function of GMM with $K$ components is

$$
P(\boldsymbol{X} ; \theta)=\sum_{k=1}^{K} \alpha_{k} \frac{1}{(2 \pi)^{\frac{n}{2}}\left|\Sigma_{k}\right|^{\frac{1}{2}}} e^{-\frac{1}{2}\left(\boldsymbol{X}-\mu_{k}\right)^{T}\left(\Sigma_{k}\right)^{-1}\left(\boldsymbol{X}-\mu_{k}\right)} .
$$

Where $\boldsymbol{X}$ is the given $n$-dimensional data, $\alpha_{k}$ is mixtu re coefficient ranging from $[0,1]$, and $\mu_{k}$ as well as $\Sigma_{k}$ are the mean and covariance matrix of $k$-th Gaussian component respectively. $\theta$ here denotes the vector of all unknown parameters. Theoretically, with enough amounts of Gaussian components, GMM can approximate any kinds of distribution at any precision.

The data obtained by the detector can be formu lated as $X=\left\{X_{1}, X_{2}, X_{3}, \ldots X_{m}\right\}$. Each $X_{i}=\left\{x_{i}, y_{i}, w_{i}, h_{i}, s_{i}\right\}$ is a 5- $d$ data point, in which $x_{i}, y_{i}$ stand for the the BB's left-top point, $w_{i}, h_{i}$ stand for the width and length of BB, and $s_{i}$ is the score for each $\mathrm{BB}$ obtained from classifier. We consider that all the BBs meet this GMM model with $k$ components, so the goal is to determine the parameters, i.e. $\alpha, \mu$ and $\Sigma$ in GMM. Given that the model now is a 5- $d$ mixture model, for each component, the parameters to be estimated are $\alpha_{k}, \mu_{k}=\left\{\mu_{x k}, \mu_{y k}, \mu_{w k}, \mu_{h k}, \mu_{s k}\right\}$ and $\Sigma_{k}$. Generally, we estimate the parameters by maximizing the following $\log$-likelihood function:

$$
L(\theta)=\sum_{i=1}^{m} \log \sum_{k=1}^{K} \alpha_{i} \frac{1}{(2 \pi)^{\frac{n}{2}}\left|\Sigma_{k}\right|^{\frac{1}{2}}} e^{-\frac{1}{2}\left(\boldsymbol{X}-\mu_{k}\right)^{T}\left(\Sigma_{k}\right)^{-1}\left(\boldsymbol{X}-\mu_{k}\right)} .
$$

\subsection{EM Optimization}

It is extremely difficult to differentiate Equation (2), make it to 0 and solves it directly mathematically. Meanwhile, data is actually incomplete since they miss their labels that tell which cluster they belong. Let latent variable $^{(15)} Z=\left\{Z_{1}, Z_{2}, Z_{3}, \ldots Z_{m}\right\}$ be the missing part of each data point and $Z_{i}$ is an $K$-dimensional vector $\left\{Z_{i}^{1}, Z_{i}^{2}, Z_{i}^{3}, \ldots Z_{i}^{K}\right\}$, where possibility $Z_{i}^{k}$ ranges from 0 to 1 depending on how much the $i$-th data belongs to $k$-th Gaussian component. is the distribution

A common way to tackle this problem is to use Expectation-Maximization (EM) algorithm ${ }^{(16)(17)}$, which is effective when latent variables are involved, to maximize Equation (2). E-step and M-step are the two parts of EM algorithm. In E-step we calculate the posterior probability of the mixture component, based on which we can determine the new parameters by M-step where the value of (2) is gradually amplified. Iterating E-step and M-step until 
convergence, the EM algorith meventually maximize (2).

E-step: In this step we denote $Q_{i}$ that means the probability of the $i$-th point is generated, or belongs to the $k$-th Gaussian component. Through Bayes' theorem, we can compute $Q_{i}$ using the estimated parameters in last iteration:

$$
\begin{aligned}
& Q_{i}\left(Z_{i}=k\right)=P\left(Z_{i}=k \mid X_{i} ; \theta\right) \\
& =\frac{\alpha_{k} \frac{1}{(2 \pi) \frac{n}{2}\left|\Sigma_{k}\right|^{\frac{1}{2}}} e^{-\frac{1}{2}\left(\boldsymbol{X}_{\boldsymbol{i}}-\mu_{k}\right)^{T}\left(\Sigma_{k}\right)^{-1}\left(\boldsymbol{X}_{\boldsymbol{i}}-\mu_{k}\right)}}{\sum_{k=1}^{K} \alpha_{j} \frac{1}{(2 \pi) \frac{n}{2}\left|\Sigma_{j}\right|^{z}} e^{-\frac{1}{2}\left(\boldsymbol{X}_{i}-\mu_{j}\right)^{T}\left(\Sigma_{j}\right)^{-1}\left(\boldsymbol{X}_{\boldsymbol{i}}-\mu_{j}\right)}} .
\end{aligned}
$$

M-step: In this step we enlarge Equation (2) by increasing the lower bound, with the help of Jensen's inequality and the derived $Q_{i}$ in the last E-step, i.e., Equation (2) is updated to

$$
\sum_{i=1}^{m} \sum_{k=1}^{K} Q_{i}\left(Z_{i}=k\right) \log \frac{\alpha_{i} \frac{1}{(2 \pi)^{\frac{n}{2}\left|\Sigma_{k}\right| \frac{1}{2}}} e^{-\frac{1}{2}\left(X_{i}-\mu_{k}\right)^{T}\left(\Sigma_{k}\right)^{-1}\left(X_{i}-\mu_{k}\right)}}{Q_{i}\left(Z_{i}=k\right)} .
$$

Taking differential with (4), we can get each parameter:

$$
\begin{gathered}
\mu_{k}=\frac{\sum_{i=1}^{m} Q_{i}\left(Z_{i}=k\right) X_{i}}{\sum_{i=1}^{m} Q_{i}\left(Z_{i}=k\right)}, \\
\Sigma_{k}=\frac{\sum_{i=1}^{m} Q_{i}\left(Z_{i}=k\right)\left(X_{i}-\mu_{k}\right)\left(X_{i}-\mu_{k}\right)^{T}}{\sum_{i=1}^{m} Q_{i}\left(Z_{i}=k\right)}, \\
\alpha_{k}=\frac{\sum_{i=1}^{m} Q_{i}\left(Z_{i}=k\right)}{m} .
\end{gathered}
$$

$\left\{\mu_{x k}, \mu_{y k}, \mu_{w k}, \mu_{h k}\right\}$ of each Gaussian component we estimate are the parameters for each result BB accordingly $\left\{x_{k}, y_{k}, w_{k}, h_{k}\right\}$.

\section{Experime nt}

In this section, we report the experimental results on merging scored bounding boxes. We compare our proposed method against several conventional approaches on different test images and classification tasks. We utilize $I o U^{(18)}$ between detection result and ground truth and its average value as the evaluation criterion.

\subsection{Setup of Experiment}

As a preparation, we put each image into a detector and get scored detections. The features and classifiers we used is list in Table 1 .

The images we use are from Penn-Fudan ${ }^{(21)}$ database and Inria ${ }^{(22)}$ person database for pedestrian detection, and Wider dataset ${ }^{(23)}$ for face detection. The experiment is carried out on 60 images for the face detection and 100 images
Table 1. Features and classifiers for different detection tasks

\begin{tabular}{|l|l|l|}
\hline & Face detection $^{(19)}$ & Pedestrian detection $^{(20)}$ \\
\hline feature & Haar-like & HOG \\
\hline classifier & Adaboost & Linear S VM \\
\hline
\end{tabular}

for the pedestrian detection, totally 160 images. The face images we choose have the following rules:

1. Faces in the images are all toward the front with as less occlusion as possible.

2. There are no hidden faces in the background

3. The faces are not blurred.

These three rules are to make sure that the detector can recognize all the faces and give scored detections as the task is not on the performance of the detector in this paper.

Given that the size of some face images in the database are too large and with a wide no-face space, we cut the redundant part without faces off (like scenery) simply for a high efficiency for detection.

Since NMS does not need to know the pre-fixed cluster number $K$ but our method needs, the $K$ we choose for GMM is the cluster number NMS returns. But due to noises, the $K$ NMS returns tends to be larger than the target number. Notice that as the GMM we use is a 5- $d$ model with score, the parameters for each Gaussian component are also supposed to be 5-dimensional. It is necessary for us to conduct NMS to the output of GMM again. Through this step, the output of GMM is refined again as the close-by low-scored results can be all discarded.

\subsection{Evaluation}

The evaluation is through intersection over union (Io $U$ ) of the ground truth $\mathrm{BB}\left(B B_{G T}\right)$ and the result $\mathrm{BB}\left(B B_{\text {result }}\right)$. $\operatorname{IoU}_{(m)}$ is the $I o U$ of the $m$-th result $\mathrm{BB}$ and its corresponding ground truth $\mathrm{BB}$.

$$
I o U_{(m)}=\frac{\operatorname{area}\left(B B_{G T}\right) \cap \operatorname{area}\left(B B_{\text {result }}\right)}{\operatorname{area}\left(B B_{G T}\right) \cup \operatorname{area}\left(B B_{\text {result }}\right)} .
$$

We divide the total 160 images into 8 groups, 5 for face detection and 3 for pedestrian detection, according to the number of target in the image. Specifically, group 1 to group 5 in face detection contain 1-2, 3-4, 5-6, 7-8, 9-10 faces respectively, and group 6 to 8 in pedestrian detection contain 1-2, 3-4, 5-6 pedestrians respectively. Each group in this paper contains 20 images.

Let $i$ be the number of image, $j$ be the group number and $N_{i j}$ is the total number of targets in the $i$-th in $j$-th group, we denote 


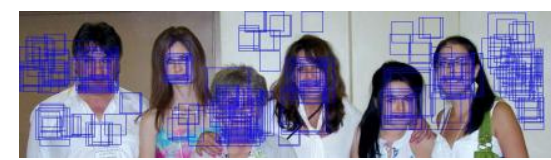

(a) Raw detections

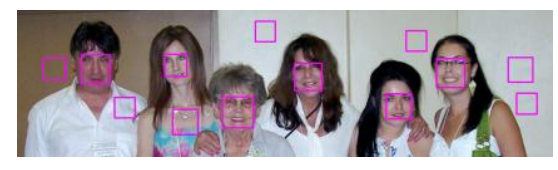

(c) K-means+NMS

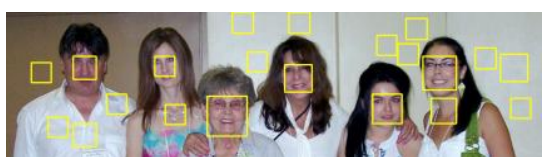

(b) NMS

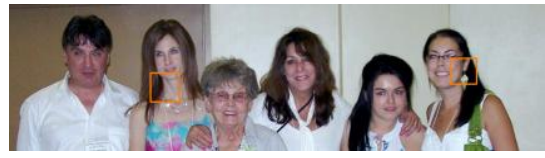

(d) Mean-shift

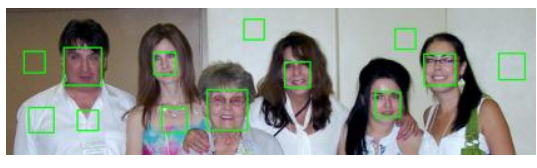

(e) GMM+NMS

Fig. 2 Example of comparative results of the image with 6 faces.

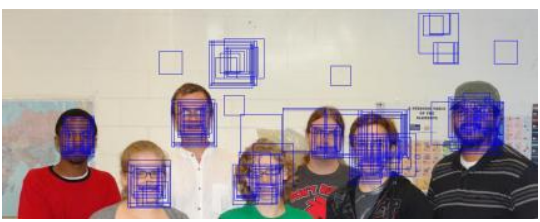

(a) Raw detections

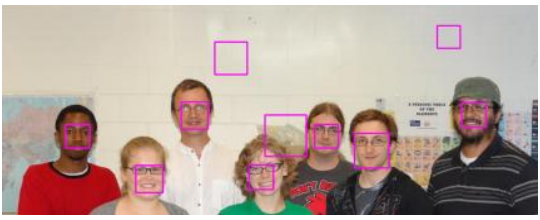

(c) K-means+NMS

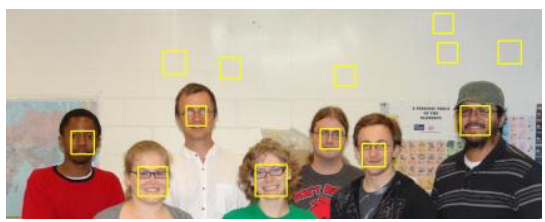

(b) NMS

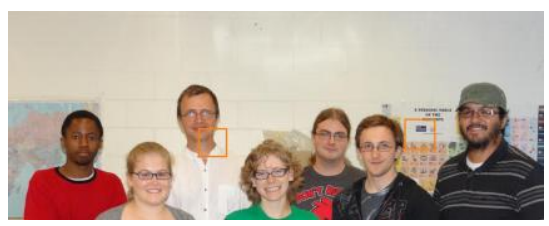

(d) Mean-shift

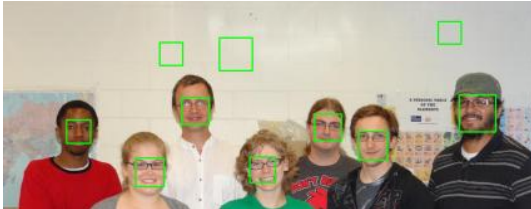

(e) GMM+NMS

Fig. 3 Example of comparative results of the image with 7 faces.

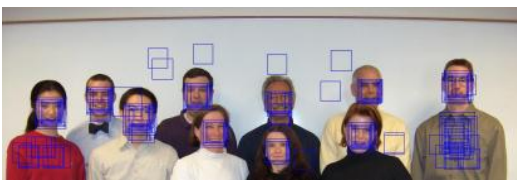

(a) Raw detections

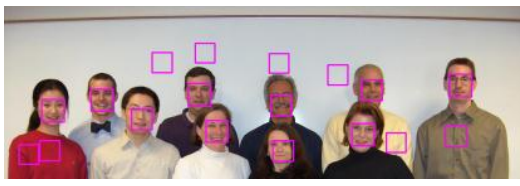

(c) K-means+NMS

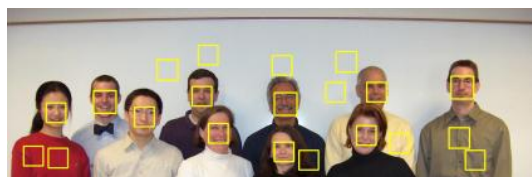

(b) NMS

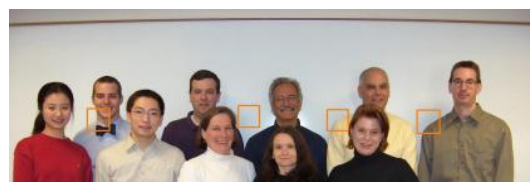

(d) Mean-shift

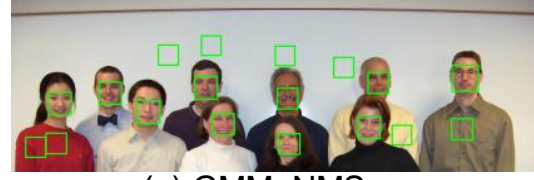

(e) GMM+NMS

Fig. 4 Example of comparative results of the image with 10 faces.

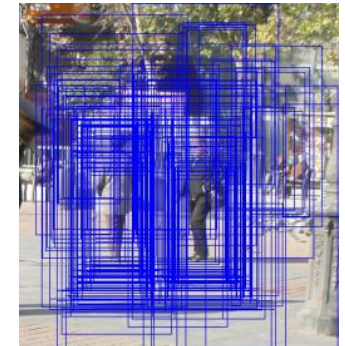

(a) Raw detections

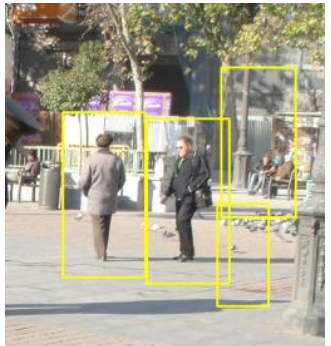

(b) NMS

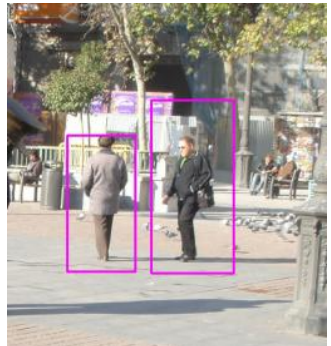

(c) K-means+NMS

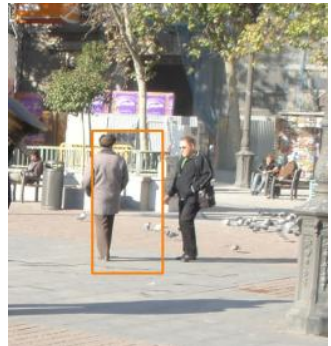

(d) Mean-shift

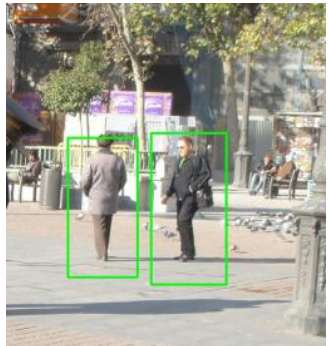

(e) GMM+NMS

Fig. 5 Example of comparative results of the image with 2 pedestrians. 


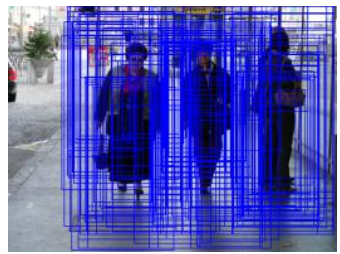

(a) Raw detections

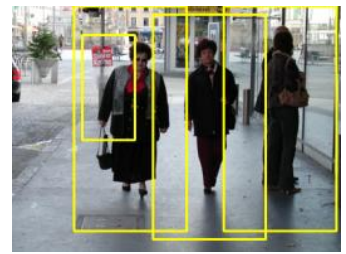

(b) NMS

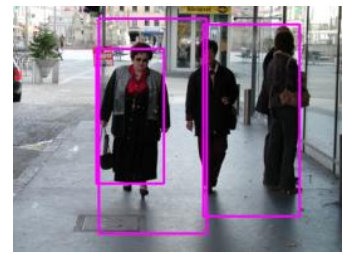

(c) K-means+NMS

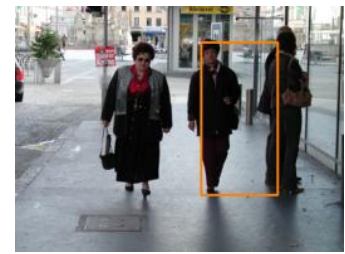

(d) Mean-shift

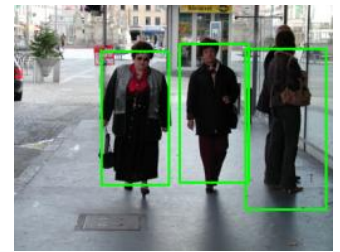

(e) GMM+NMS

Fig. 6 Example of comparative results of the image with 3 pedestrians.

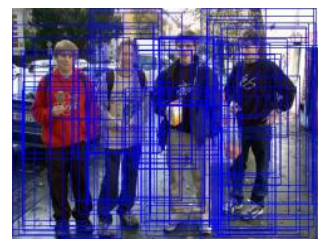

(a) Raw detections

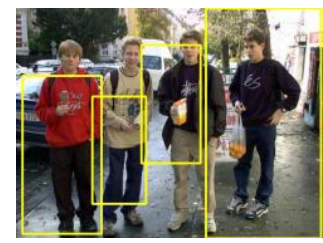

(b) NMS

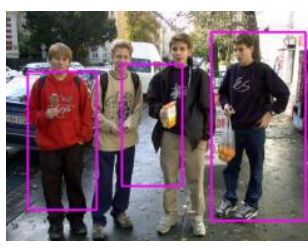

(c) K-means+NMS

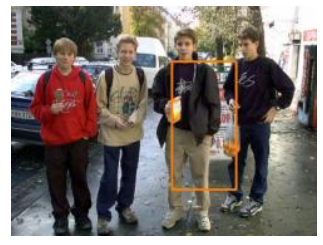

(d) Mean-shift

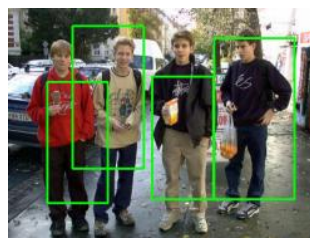

(e) GMM+NMS

Fig. 7 Example of comparative results of the image with 4 pedestrians.

$$
a_{i j}=\frac{\sum_{m=1}^{N_{i j}} \operatorname{IoU}_{(m)}}{N_{i j}} .
$$

as the average $I o U$ of the $i$-th image in $j$-th group.

Also, we denote

$$
a_{j}=\frac{\sum_{i=1}^{20} a_{i j}}{20} .
$$

as the average $I o U$ of the $j$-th group.

Table 2 shows $a_{j}$ in Equation (10) for $j=1,2,3,4,5$ in column "face detection" and $j=6,7,8$ in column "pedestrian detection". $a_{j}$ both in "Face detection" and "Pedestrian detection" is increased with the help of the combination of GMM and NMS.

There exists a possibility that several result BBs have intersection with the same ground truth $\mathrm{BB}$. In this paper, we only take the $\mathrm{BB}$ that has the $\max I o U$ with the ground truth $\mathrm{BB}$ among all the intersecting result BBs.

Also, one result $\mathrm{BB}$ may have intersection with more than one ground truth $\mathrm{BB}$ at the same time especially when two ground truth BBs are relatively close. If we face this situation, we take the result $\mathrm{BB}$ as the result of the ground truth $\mathrm{BB}$ with which the $I o U$ is the highest, and one result $\mathrm{BB}$ can only be calculated once, one ground truth $\mathrm{BB}$ also only can have one result $\mathrm{BB}$ as the result.

Fig.2-Fig.7 are some comparative results by the conventional approaches and proposed approach. In Fig.2-Fig.4, we can find that the traditional NMS tends to select the BB that only contains partial face regardless of other better BBs with lower scores in some case, and the same for the pedestrian detection task in Fig.5-Fig.7, which is the major disadvantage mentioned before that NMS only chooses the given top-scored detection as the output. Instead, GMM can make a comprehensive decision from judging the label of each detection to find the center of each cluster, avoiding the influence of true positives.

Nevertheless both conventional approaches and proposed approach have not shown good results in some cases, such as the second face from left in Fig.3, or the first person

\begin{tabular}{|c|c|c|c|c|c|c|}
\hline Detection task & Number of targets & Group number $j$ & NMS & Mean-shift & $K$-means+NMS & GMM+NMS \\
\hline \multirow{5}{*}{$\begin{array}{c}\text { Face } \\
\text { Detection }\end{array}$} & (1-2 faces) $\times 20$ images & 1 & 0.45 & 0.45 & 0.48 & 0.50 \\
\hline & (3-4 faces) $\times 20$ images & 2 & 0.49 & 0.26 & 0.50 & 0.54 \\
\hline & (5- 6 faces) $\times 20$ images & 3 & 0.44 & 0.04 & 0.47 & 0.52 \\
\hline & (7-8 faces) $\times 20$ images & 4 & 0.46 & 0.05 & 0.51 & 0.56 \\
\hline & $(9-10$ faces $) \times 20$ images & 5 & 0.47 & 0.03 & 0.50 & 0.55 \\
\hline \multirow{3}{*}{$\begin{array}{l}\text { Pedestrian } \\
\text { Detection }\end{array}$} & ( $1-2$ pedestrians $) \times 20$ images & 6 & 0.43 & 0.42 & 0.46 & 0.57 \\
\hline & (3-4 pedestrians) $\times 20$ images & 7 & 0.37 & 0.31 & 0.35 & 0.45 \\
\hline & (5-6 pedestrians) $\times 20$ images & 8 & 0.39 & 0.21 & 0.35 & 0.44 \\
\hline
\end{tabular}

Table 2. $a_{j}$.in Equation (10). Average $I o U$ for each image in each group $a_{i j}$ is used to calculate the $a_{j}$. 
in Fig.7. One possible reason assumed is that the detections themselves are not sufficient and satisfactory that GMM cannot work well. Another explanation is that since GMM is based on the assumption that the data complies with the Gaussian distribution, the limitation can appear when the data does not follow the Gaussian distribution very well.

\section{Conclusion}

In this paper, we propose a new approach to merge scored bounding boxes for object detection with the combination of Gaussian Mixture Model and non-maximum suppression. The results of the experiment on 160 images with different sizes of faces and pedestrians show that the $I o U$ of final detections increased by $7.8 \%$ by average comparing with NMS. However, as the future work, the proposed method still has a lot of improvable parts. For example, a more powerful GMM model such as generalized GMM can be applied to achieve better performance.

\section{References}

(1) Viola, Paul, and Michael J. Jones : "Robust real-time face detection", International journal of computer vision, Vol.57, No.2, pp. 137-154, 2004

(2) Ouyang, Wanli, and Xiaogang Wang : "Single-pedestrian detection aided by multi-pedestrian detection", Computer Vision and Pattern Recognition (CVPR), pp. 3198-3205, 2013

(3) Ren, Shaoqing, et al : "Faster r-cnn: Towards real-time object detection with region proposal networks", Advances in neural information processing systems, pp. 91-99, 2015

(4) Hosang, Jan, Rodrigo Benenson, and Bernt Schiele : "Learning non-maximum suppression", Computer Vision and Pattern Recognition (CVPR), 2017

(5) Steinbach, Michael, George Karypis, and Vip in Kumar : "A comparison of document clustering techniques", KDD workshop on text mining, Vol. 400, No. 1, pp. 525-526, 2000

(6) Lu, Xuequan, et al : "GPF: GMM-inspired Feature-preserving Point Set Filtering", Journal of IEEE Transactions on Visualization and Computer Graphics (TVCG), 2017

(7) Zivkovic, Zoran : "Improved adaptive Gaussian mixture model for background subtraction", 17th International Conference on Computer Vision (ICCV), Vol. 2, pp. 28-31, 2004
(8) Cheng, Yizong : "Mean shift, mode seeking, and clustering", IEEE transactions on pattern analysis and machine intelligence (TPAMI), Vol.17, No.8, pp.790-799, 1995

(9) Comaniciu, Dorin, and Peter Meer : "Mean shift: A robust approach toward feature space analysis", IEEE Transactions on pattern analys is and machine intelligence (TPAMI), Vol.24, No.5, pp.603-619, 2002

(10) Georgescu, Bogdan, Ilan Shimshoni, and Peter Meer : "Mean Shift Based Clustering in High Dimensions: A Texture Classification Example", International Conference on Computer Vision (ICCV), Vol. 3, pp.456, 2003

(11) WAGSTAFF, Kiri, et al : "Constrained k-means clustering with background knowledge", The International on Machine Learning (ICML), Vol.1, pp. 577-584, 2001

(12) Likas, Aristidis, Nikos Vlassis, and Jakob J. Verbeek : "The global k-means clustering algorithm", journal of Pattern recognition, Vol.36, No.2, pp. 451-461, 2003

(13) Wang, Jinjun, et al : "Locality-constrained linear coding for image classification", Computer Vision and Pattern Recognition (CVPR), 2010 IEEE Conference on. IEEE, pp.3360-3367, 2010

(14) Wojcikiewicz, W.: "Probabilistic modeling of multiple observations in face detection", Technical report, Hu mboldt-Universit"at zu Berlin, 2008

(15) Borsboom, Denny, Gideon J. Mellenbergh, and Jaap Van Heerden. "The theoretical status of latent variables", Journal of Psychological review, Vol.110, No.2, pp. 203, 2003

(16) Dempster, Arthur P., Nan M. Laird, and Donald B. Rubin : "Maximum likelihood from incomplete data via the EM algorithm", Journal of the royal statistical society. Series B (methodological), pp. 1-38, 1977

(17) Bock, R. Darrell, and Murray Aitkin : "Marginal maximu m likelihood estimation of item parameters: Application of an EM algorithm", Journal of the Psychometrika, Vol.46, No.4, pp. 443-459, 1981

(18) Ahmed, Faruk, Dany Tarlow, and Dhruv Batra : "Optimizing expected intersection-over-union with candidate-constrained crfs", The IEEE International Conference on Computer Vision (ICCV), 2015

(19) Viola, Paul, and Michael Jones : "Rapid object detection using a boosted cascade of simple features" Computer Vision and Pattern Recognition (CVPR), Vol. 1, 2001

(20) Dalal, Navneet, and Bill Triggs : "Histograms of 
oriented gradients for human detection", Computer Vision and Pattern Recognition (CVPR), Vol. 1, pp. 886-893, 2005

(21) Liming Wang, Jianbo Shi, Gang Song, I-fan Shen: Object Detection Combining Recognition and Segmentation", Asian Conference on Computer Vision (ACCV), pp. 189-199, 2007

(22) Wang, Xiaoyu, Tony X. Han, and Shuicheng Yan: "An HOG-LBP human detector with partial occlusion handling", International Conference on Computer Vision (ICCV), pp. 32-39, 2009

(23) YANG, Shuo, et al : "Wider face : A face detection benchmark", In: Proceedings of the IEEE Conference on Computer Vision and Pattern Recognition(CVPR). pp. 5525-5533, 2016 